$03,04,19$

\title{
Синтез, кристаллическая структура и теплофизические свойства замещенных титанатов $\mathrm{Bi}_{2} \mathrm{Pr}_{2} \mathrm{Ti}_{3} \mathrm{O}_{12}$ и $\mathrm{Bi}_{2} \mathrm{Nd}_{2} \mathrm{Ti}_{3} \mathrm{O}_{12}$
}

\author{
( Л.Т. Денисова ${ }^{1}$, М.С. Молокеев ${ }^{1,2}$, Н.А. Галиахметова ${ }^{1}$, В.М. Денисов ${ }^{1}$, Г.В. Васильев ${ }^{1}$ \\ ${ }^{1}$ Сибирский фредеральный университет, \\ Красноярск, Россия \\ ${ }^{2}$ Институт фризики им. Л.В. Киренского ФИЦ КНЦ СО РАН, \\ Красноярск, Россия \\ E-mail: Idenisova@sfu-kras.ru
}

Поступила в Редакцию 31 марта 2021 г.

В окончательной редакции 3 апреля 2021 г.

Принята к публикации 3 апреля 2021 г.

Титанаты $\mathrm{Bi}_{2} \mathrm{Pr}_{2} \mathrm{Ti}_{3} \mathrm{O}_{12}$ и $\mathrm{Bi}_{2} \mathrm{Nd}_{2} \mathrm{Ti}_{3} \mathrm{O}_{12}$ получены методом твердофазного синтеза последовательным обжигом на воздухе при температурах $1003-1323 \mathrm{~K}$ стехиометрических смесей $\mathrm{Bi}_{2} \mathrm{O}_{3}, \mathrm{Nd}_{2} \mathrm{O}_{3}, \operatorname{Pr}_{6} \mathrm{O}_{11}$ и $\mathrm{TiO}_{2}$. Методом рентгеновской дифракции определена их кристаллическая структура. Высокотемпературная теплоемкость определена с использованием дифференциальной сканирующей калориметрии. На основании экспериментальных данных $C_{p}=f(T)$ рассчитаны основные термодинамические функции.

Ключевые слова: замещенные титанаты висмута, твердофазный синтез, кристаллическая структура, высокотемпературная теплоемкость, термодинамические функции.

DOI: 10.21883/FTT.2021.08.51153.070

\section{1. Введение}

Замещением части висмута в титанате $\mathrm{Bi}_{4} \mathrm{Ti}_{3} \mathrm{O}_{12}$ на редкоземельные элементы получаются твердые растворы $\mathrm{Bi}_{4-x} R_{x} \mathrm{Ti}_{3} \mathrm{O}_{12}(R=\mathrm{P} 3 Э)$. В последнее время к таким материалам наблюдается устойчивый интерес. Были получены твердые растворы содержащие La [1-3], $\mathrm{Ce}[2], \mathrm{Nd}$ [4-6], Sm [7-9], Eu [10], Er [11], Pr, Nd, $\mathrm{Cd}$, Dy $[12,13]$. Такое внимание к твердым растворам на основе слоистого титаната висмута связано с тем, что замещение висмута на редкоземельные элементы изменяет свойства титаната (диэлектрические константы, проводимость, температура Кюри и т.д.). Согласно $[3,14,15]$, замещение $\mathrm{Bi}$ на $\mathrm{La}$ уменьшает усталость $\mathrm{Bi}_{4} \mathrm{Ti}_{3} \mathrm{O}_{12}$, а также снижает поляризацию. Считается, что такие материалы по ряду функциональных характеристик смогут превосходить такой известный сегнетоэлектрик как цирконат свинца [3]. Большинство работ, посвященных замещенному титанату висмута, связаны с изучением их электрофизических свойств. Последнее связано с перспективностью их использования в акусто- и оптоэлектронике, пьезоэлектрических преобразователях, сегнетоэлектрической памяти и т.д. Тем не менее, не смотря на такое внимание к этим материалам, многие их свойства к настоящему времени не исследованы. В первую очередь это касается теплофизических характеристик. Некоторые из имеющихся данных о кристаллической структуре твердых растворов не согласуются между собой. Так, например, в работах $[13,14]$ сообщено, что при замещении Вi на РЗЭ сохраняется орторомбическая симметрия, тогда как авторы $[3,16]$ утверждают, что она изменяется на тетрагональную. Диаграммы состояния систем $\mathrm{Bi}_{4} \mathrm{Ti}_{3} \mathrm{O}_{12}-R_{4} \mathrm{Ti}_{3} \mathrm{O}_{12}$ не построены. Заметим, что на диаграммах состояния $\mathrm{Nd}_{2} \mathrm{O}_{3}-\mathrm{TiO}_{2}$ [17], $\mathrm{Er}_{2} \mathrm{O}_{3}-\mathrm{TiO}_{2}$ [18], $\mathrm{Lu}_{2} \mathrm{O}_{3}-\mathrm{TiO}_{2}$ [19], $\quad \mathrm{Bi}_{2} \mathrm{O}_{3}-\mathrm{TiO}_{2}-\mathrm{Y}_{2} \mathrm{O}_{3} \quad$ [20], $\mathrm{La}_{2} \mathrm{O}_{3}-$ $\mathrm{TiO}_{2}[21], \quad \mathrm{Gd}_{2} \mathrm{O}_{3}\left(\mathrm{Dy}_{2} \mathrm{O}_{3}\right)-\mathrm{TiO}_{2} \quad[22], \quad \mathrm{R}_{2} \mathrm{O}_{3}-\mathrm{TiO}_{2}$ $(\mathrm{R}=\mathrm{La}, \mathrm{Ce}, \mathrm{Pr}, \mathrm{Nd}$, Sm, Gd, Tb, Dy, Ho, Er, Yb, Y) [23] наличие соединений типа $R_{4} \mathrm{Ti}_{3} \mathrm{O}_{12}$, не отмечено. Тем не менее, имеются данные об их образовании: $\mathrm{Eu}_{4} \mathrm{Ti}_{3} \mathrm{O}_{12}$ [23], $\mathrm{La}_{4} \mathrm{Ti}_{3} \mathrm{O}_{12}$ [24,25], $\mathrm{Nd}_{4} \mathrm{Ti}_{3} \mathrm{O}_{12}$ [17]. Для компьютерного моделирования этих систем необходимы данные о термодинамических свойствах образующихся соединений. Такие сведения в литературе (за исключением простых оксидов и $\mathrm{Bi}_{4} \mathrm{Ti}_{3} \mathrm{O}_{12}$ ) отсутствуют.

В настоящей работе представлены результаты синтеза, исследований кристаллической структуры и теплофизических свойств титанатов $\mathrm{Bi}_{2} \mathrm{Pr}_{2} \mathrm{Ti}_{3} \mathrm{O}_{12}$ и $\mathrm{Bi}_{2} \mathrm{Nd}_{2} \mathrm{Ti}_{3} \mathrm{O}_{12}$.

\section{2. Синтез образцов и техника эксперимента}

Замещенные титанаты $\mathrm{Bi}_{2} \mathrm{Pr}_{2} \mathrm{Ti}_{3} \mathrm{O}_{12}$ и $\mathrm{Bi}_{2} \mathrm{Nd}_{2} \mathrm{Ti}_{3} \mathrm{O}_{12}$ получены твердофазным синтезом из исходных оксидов $\mathrm{Bi}_{2} \mathrm{O}_{3}, \mathrm{Nd}_{2} \mathrm{O}_{3}, \mathrm{TiO}_{2}$ (ос.ч) и $\mathrm{Pr}_{6} \mathrm{O}_{11}$ (Alfa Aesor, 99.999\%). Для этого стехиометрические смеси предварительно прокаленных оксидов гомогенизировали в планетарной шаровой мельнице PM 100 фирмы Retsch (Германия) со стаканами и шарами из диоксида циркония (скорость вращения рабочих сосудов $180 \mathrm{rev} / \mathrm{min}$, а продолжительность обработки $30 \mathrm{~min}$ ). Принимая во внимание, что после такой обработки полученные порошки могут взаимодействовать с окружающей средой [26], их сразу помещали в полиэтиленовые контейнеры, вакуумиро- 
вали и запаивали. После этого прессовали при помощи изостатического пресса YLJ-CIP-20B $(P=150 \mathrm{MPa}$, $\tau=5 \mathrm{~min})$. Полученные образцы обжигали на воздухе по $10 \mathrm{~h}$ при температурах 1073, 1103, 1153, 1273, $1323 \mathrm{~K}$ $(1203 \mathrm{~K}-20 \mathrm{~h})$. После каждой температуры их перетирали в планетарной мельнице и снова прессовали при тех же условиях. Порошковые рентгенограммы титанатов $\mathrm{Bi}_{2} \mathrm{Nd}_{2} \mathrm{Ti}_{3} \mathrm{O}_{12}$ и $\mathrm{Bi}_{2} \mathrm{Pr}_{2} \mathrm{Ti}_{3} \mathrm{O}_{12}$ снимали при комнатной температуре на дифрактометре D8 ADVANCE фирмы Bruker $\left(\mathrm{Cu} K_{\alpha}\right.$-излучение) с использованием линейного детектора VANTEC.

Теплоемкость титанатов $\mathrm{Bi}_{2} \mathrm{Pr}_{2} \mathrm{Ti}_{3} \mathrm{O}_{12}$ и $\mathrm{Bi}_{2} \mathrm{Nd}_{2} \mathrm{Ti}_{3} \mathrm{O}_{12}$ измеряли методом дифференциальной сканирующей калориметрии с использованием термоанализатора STA 449 C Jupiter (NETZSCH, Герания). Методика экспериментов аналогична описанной ранее $[27,28]$. Ошибка экспериментов не превышала $2 \%$.

\section{3. Экспериментальные результаты}

Bсе рефлексы на рентгенограммах были проиндицированы тетрагональной ячейкой $P 4_{2} / n m c$. Поэтому эта структура была взята в качестве исходной модели для уточнения методом Ритвельда в программе TOPAS 4.2 [29]. В независимой части ячейки есть две позиции $\mathrm{Bi}$, и обе они были заселены ионами $\mathrm{Bi} / \mathrm{Pr}$ и $\mathrm{Bi} / \mathrm{Nd}$ для каждой фазы соответственно. Заселенности позиций уточнялись и для повышения стабильности уточнения на сумму количества ионов $\mathrm{Bi}$ и $\operatorname{Pr}(\mathrm{Nd})$ в ячейке наложено ограничение в виде линейных уравнений. В результате уточнения всех структур прошли стабильно и дали низкие факторы недостоверности (табл. 1). Координаты атомов и тепловые параметры представлены в табл. 2, a основные длины связей - в табл. 3, разностные рентгенограммы на рис. 1 .

Структуру незамещенного титаната висмута исследовали неоднократно. При этом были получены проти-

Таблица 1. Основные параметры экспериментов и результаты уточнения $\mathrm{Bi}_{2} \mathrm{Pr}_{2} \mathrm{Ti}_{3} \mathrm{O}_{12}$ и $\mathrm{Bi}_{2} \mathrm{Nd}_{2} \mathrm{Ti}_{3} \mathrm{O}_{12}$

\begin{tabular}{c|c|c}
\hline Состав & $\mathrm{Bi}_{2} \mathrm{Pr}_{2} \mathrm{Ti}_{3} \mathrm{O}_{12}$ & $\mathrm{Bi}_{2} \mathrm{Nd}_{2} \mathrm{Ti}_{3} \mathrm{O}_{12}$ \\
\hline Пр. гр. & $P 4_{2} / n m c$ & $P 4_{2} / n m c$ \\
$a, \AA$ & $3.81590(11)$ & $3.80943(8)$ \\
$c, \AA$ & $32.7879(15)$ & $32.7697(11)$ \\
$V, \mathrm{a}^{3}$ & $477.43(4)$ & $475.55(3)$ \\
$Z$ & 2 & 2 \\
$2 \theta$ интервал, & $5-120$ & $5-120$ \\
$R_{w p}, \%$ & 7.91 & 6.75 \\
$R_{p}, \%$ & 6.31 & 5.35 \\
$R_{B}, \%$ & 2.50 & 1.69 \\
$\chi^{2}$ & 1.85 & 1.68
\end{tabular}

Пр и м е ч а и е. $a, c-$ параметры ячейки; $V-$ объем ячейки; факторы недостоверности: $R_{w p}-$ весовой профильный, $R_{p}-$ профильный, $R_{B}$ - интегральный; $\chi^{2}-$ качество подгонки.
Таблица 2. Координаты атомов и изотропные тепловые параметры структур твердых растворов $\mathrm{Bi}_{2} \mathrm{Pr}_{2} \mathrm{Ti}_{3} \mathrm{O}_{12}$ и $\mathrm{Bi}_{2} \mathrm{Nd}_{2} \mathrm{Ti}_{3} \mathrm{O}_{12}$

\begin{tabular}{c|c|c|l|l|c}
\hline Aтом & $x$ & $y$ & \multicolumn{1}{c}{$z$} & \multicolumn{1}{c}{$B_{\text {iso }}$} & Occ \\
\hline \multicolumn{5}{c}{$\mathrm{Bi}_{2} \operatorname{Pr}_{2} \mathrm{Ti}_{3} \mathrm{O}_{12}$} \\
\hline Bi1 & 0.25 & 0.25 & $0.31600(8)$ & $0.55(11)$ & $0.288(8)$ \\
Pr1 & 0.25 & 0.25 & $0.31600(8)$ & $0.55(11)$ & $0.712(8)$ \\
Bi2 & 0.25 & 0.25 & $0.46124(7)$ & $0.30(10)$ & $0.701(8)$ \\
Pr2 & 0.25 & 0.25 & $0.46124(7)$ & $0.30(10)$ & $0.299(8)$ \\
Ti1 & 0.25 & 0.25 & 0.75 & $0.5(3)$ & 1 \\
Ti2 & 0.25 & 0.25 & $0.6209(2)$ & $0.50(19)$ & 1 \\
O1 & 0.75 & 0.75 & $0.2375(10)$ & $1.1(3)$ & 1 \\
O2 & 0.75 & 0.75 & $0.4940(18)$ & $1.1(3)$ & 1 \\
O3 & 0.25 & 0.25 & $0.6899(7)$ & $1.1(3)$ & 1 \\
O4 & 0.25 & 0.25 & $0.5769(9)$ & $1.1(3)$ & 1 \\
O5 & 0.75 & 0.75 & $0.3556(15)$ & $1.1(3)$ & 1 \\
O6 & 0.75 & 0.75 & $0.1359(17)$ & $1.1(3)$ & 1 \\
\hline \multicolumn{5}{|c}{$\mathrm{Bi}_{2} \mathrm{Nd}_{2} \mathrm{Ti}_{3} \mathrm{O}_{12}$} \\
Bi1 & 0.25 & 0.75 & $0.31594(7)$ & $0.46(11)$ & $0.215(6)$ \\
Pr1 & 0.25 & 0.75 & $0.31594(7)$ & $0.46(11)$ & $0.785(6)$ \\
Bi2 & 0.25 & 0.75 & $0.46134(6)$ & $0.30(10)$ & $0.777(7)$ \\
Pr2 & 0.25 & 0.75 & $0.46234(6)$ & $0.30(10)$ & $0.223(7)$ \\
Ti1 & 0.25 & 0.75 & 0.75 & $0.5(3)$ & 1 \\
Ti2 & 0.25 & 0.75 & $0.6217(2)$ & $0.50(18)$ & 1 \\
O1 & 0.75 & 0.75 & $0.2376(9)$ & $1.3(3)$ & 1 \\
O2 & 0.75 & 0.75 & $0.4954(18)$ & $1.3(3)$ & 1 \\
O3 & 0.25 & 0.75 & $0.6911(5)$ & $1.3(3)$ & 1 \\
O4 & 0.25 & 0.75 & $0.5713(7)$ & $1.3(3)$ & 1 \\
O5 & 0.75 & 0.75 & $0.3569(13)$ & $1.3(3)$ & 1 \\
O6 & 0.75 & 0.75 & $0.1353(15)$ & $1.3(3)$ & 1 \\
& &
\end{tabular}

Таблица 3. Основные длины связей $(\AA)$

\begin{tabular}{l|l|l|l}
\hline \multicolumn{4}{c}{$\mathrm{Bi}_{2} \mathrm{Pr}_{2} \mathrm{Ti}_{3} \mathrm{O}_{12}$} \\
\hline$(\mathrm{Bi} 1 / \mathrm{Pr} 1)-\mathrm{O}^{i}$ & $2.59(2)$ & $\mathrm{Ti} 1-\mathrm{O} 1^{i i i}$ & $1.952(7)$ \\
$(\mathrm{Bi} 1 / \mathrm{Pr} 1)-\mathrm{O}^{i i}$ & $2.7051(16)$ & $\mathrm{Ti} 1-\mathrm{O} 3$ & $1.97(2)$ \\
$(\mathrm{Bi} 1 / \mathrm{Pr} 1)-\mathrm{O} 5$ & $2.31(3)$ & $\mathrm{Ti} 2-\mathrm{O} 3$ & $2.26(2)$ \\
$(\mathrm{Bi} 1 / \mathrm{Pr} 1)-\mathrm{O}^{i}$ & $2.84(4)$ & $\mathrm{Ti} 2-\mathrm{O} 4$ & $1.44(3)$ \\
$(\mathrm{Bi} 2 / \mathrm{Pr} 2)-\mathrm{O} 2$ & $2.19(3)$ & $\mathrm{Ti} 2-\mathrm{O} 5^{i i i}$ & $2.058(19)$ \\
$(\mathrm{Bi} 2 / \mathrm{Pr} 2)-\mathrm{O}^{i i i}$ & $2.41(4)$ & $\mathrm{Ti} 2-\mathrm{O6}^{i v}$ & $1.970(14)$ \\
$(\mathrm{Bi} 2 / \mathrm{Pr} 2)-4 \mathrm{O}^{i i}$ & $2.974(12)$ & & \\
\hline \multicolumn{4}{|c}{$\mathrm{Bi}_{2} \mathrm{Nd}_{2} \mathrm{Ti}_{3} \mathrm{O}_{12}$} \\
\hline$(\mathrm{Bi} 1 / \mathrm{Nd} 1)-\mathrm{O}^{i}$ & $2.589(19)$ & $\mathrm{Ti} 1-\mathrm{O} 1^{i i i}$ & $1.948(6)$ \\
$(\mathrm{Bi} 1 / \mathrm{Nd} 1)-\mathrm{O}^{i i}$ & $2.7036(15)$ & $\mathrm{Ti} 1-\mathrm{O} 3$ & $1.930(18)$ \\
$(\mathrm{Bi} 1 / \mathrm{Nd} 1)-\mathrm{O}^{i}$ & $2.33(3)$ & $\mathrm{Ti} 2-\mathrm{O} 3$ & $2.274(19)$ \\
$(\mathrm{Bi} 1 / \mathrm{Nd} 1)-\mathrm{O}^{i}$ & $2.49(3)$ & $\mathrm{Ti} 2-\mathrm{O} 4$ & $1.65(2)$ \\
$(\mathrm{Bi} 2 / \mathrm{Nd} 2)-\mathrm{O} 2$ & $2.21(3)$ & $\mathrm{Ti} 2-\mathrm{O} 5^{i i i}$ & $2.029(15)$ \\
$(\mathrm{Bi} 2 / \mathrm{Nd} 2)-\mathrm{O} 2^{i i i}$ & $2.37(4)$ & $\mathrm{Ti} 2-\mathrm{O} 6^{i v}$ & $1.956(11)$ \\
$(\mathrm{Bi} 2 / \mathrm{Nd} 2)-4 \mathrm{O}^{i i}$ & $2.899(9)$ & &
\end{tabular}

Пр им е чан и е. Элементы симметрии: $(i)-x+1,-y+1,-z+1 / 2$; (ii) $-x,-y+1,-z+1 ;$ (iii) $-x+1,-y+1,-z+1$; (iv) $x+3 / 2,-y+1 / 2, z+1 / 2 ;(v)-x+2,-y+1,-z+1 / 2$.

воречивые результаты. Так, например, согласно [11,30], $\mathrm{Bi}_{4} \mathrm{Ti}_{3} \mathrm{O}_{12}$ при комнатной температуре имеет ромбическую элементарную ячейку (пр.гр. Fmmm). Боль- 

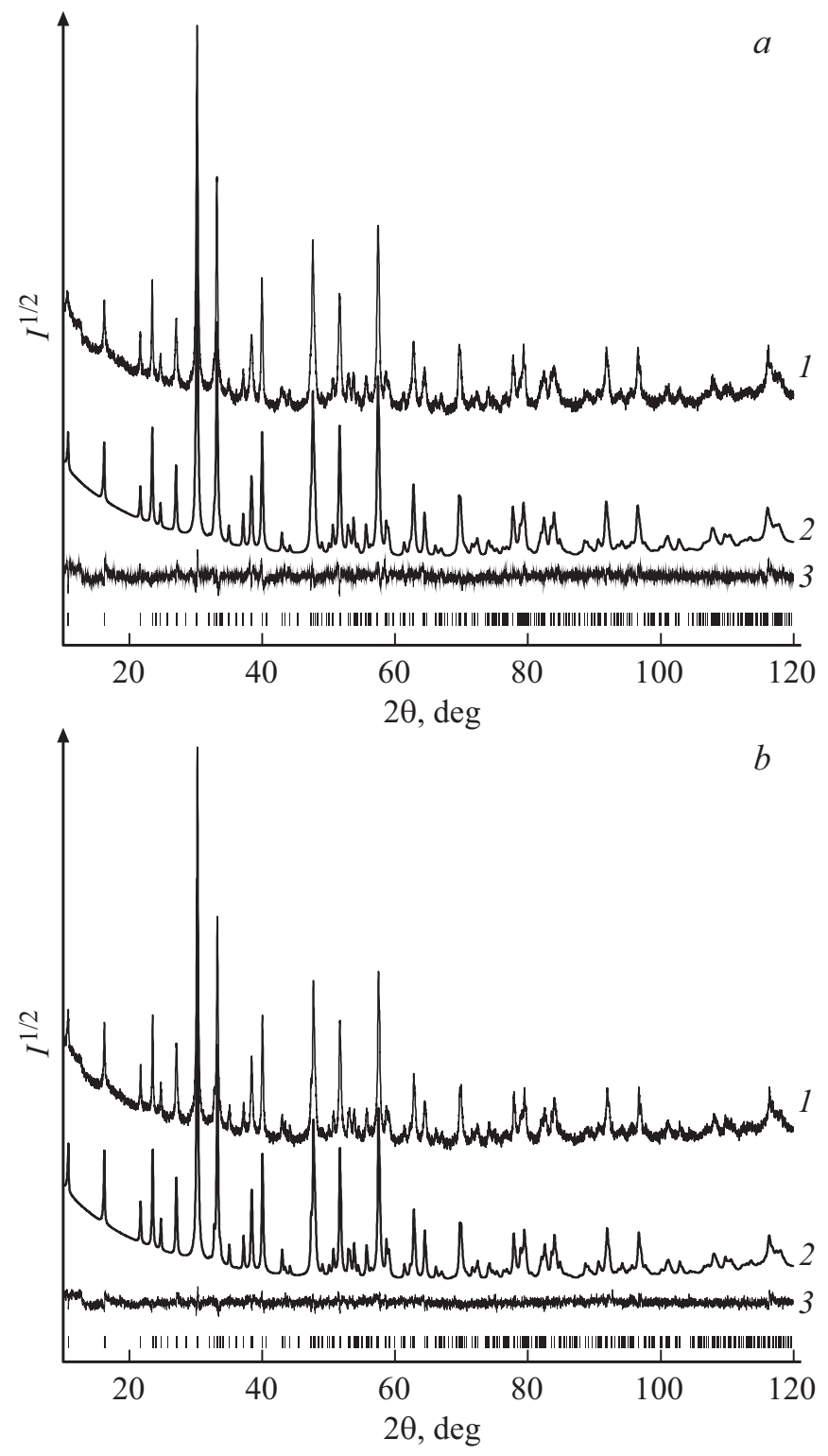

Рис. 1. Экспериментальный (1), расчетный (2) и разностный (3) профили рентгенограмм $\mathrm{Bi}_{2} \mathrm{Pr}_{2} \mathrm{Ti}_{3} \mathrm{O}_{12} \quad(a)$ и $\mathrm{Bi}_{2} \mathrm{Nd}_{2} \mathrm{Ti}_{3} \mathrm{O}_{12}(b)$ после уточнения методом минимизации производной разности; штрихи указывают расчетные положение рефлексов.

шинство авторов считают, что для этого титаната характерна пр.гр. $B 2 c b[13,14,16]$ или $A b a 2[31,32]$ (получено трансформированием из $B 2 c b$ (Aba2: $a b c=$ $\left.\left.=B 2 c b: b^{\prime} c^{\prime} a^{\prime}[31]\right)\right)$. По нашим данным $\mathrm{Bi}_{4} \mathrm{Ti}_{3} \mathrm{O}_{12}$ имеет пр.гр. Aba2. Из этих данных и табл. 1 следует, что при замещении $\mathrm{Bi}$ на $\operatorname{Pr}$ или $\mathrm{Nd}$ происходит изменение структуры (пр.гр. $P 4_{2} / n m c$ ). По данным работы авторов [33], соединения $\mathrm{Bi}_{2} R_{2} \mathrm{Ti}_{3} \mathrm{O}_{12}(R=\mathrm{La}, \mathrm{Pr}, \mathrm{Nd}$, $\mathrm{Sm})$ имеют пр.гр. I4/mmm со значениями параметров элементарной ячейки $a \sim 3.8 \AA$ и $c \sim 33 \AA$. Подобное отмечено для $\mathrm{Bi}_{2} \mathrm{Nd}_{2} \mathrm{Ti}_{3} \mathrm{O}_{12}$ [16] и $\mathrm{Bi}_{2} \mathrm{La}_{2} \mathrm{Ti}_{3} \mathrm{O}_{12}$ [34]. В то же время имеются и другие сведения. Согласно [35], при проведении реакции между $\mathrm{K}_{2} \mathrm{La}_{2} \mathrm{Ti}_{3} \mathrm{O}_{10}$ и $\mathrm{BiOCl}$ образующийся $\mathrm{Bi}_{2} \mathrm{La}_{2} \mathrm{Ti}_{3} \mathrm{O}_{12}$ имеет орторомбическую элементарную ячейку ( $a=5.441(1) \AA, b=5.399(1) \AA$, $c=32.944(4) \AA)$.

Проведенный нами поиск подходящей фазы, связанной с низкосимметричной Aba2 групповыми свойствами, который был проведен в программе PSEUDO [36], показал, что наиболее подходящими структурами (смещение атомов меньше $1 \AA$ ) могут быть две структуры с пространственными группами $P 4_{2} / n m c$ и $I 4 / \mathrm{mmm}$. Тестовое уточнение для обоих моделей дало брэгговские $R$-факторы для $\mathrm{Bi}_{2} \mathrm{Pr}_{2} \mathrm{Ti}_{3} \mathrm{O}_{12}$ и $\mathrm{Bi}_{2} \mathrm{Nd}_{2} \mathrm{Ti}_{3} \mathrm{O}_{12} \quad 2.50 \%$ и $1.69 \% \quad\left(P 4_{2} / n m c\right), 3.91 \%$ и $3.49 \%$ (I4/ $\left.\mathrm{mmm}\right)$ соответственно. Учитывая то, что эти значения для модели $P 4_{2} / n m c$ оказались заметно меньше, а так же то, что число уточняемых структурных параметров у двух моделей близко (8 уточняемых координат у $I 4 / \mathrm{mmm}$ и 9 координат у $\left.\mathrm{P} 4_{2} / \mathrm{nmc}\right)$, и то, что структура $I 4 / \mathrm{mmm}$ имела большие тепловые параметры у всех атомов кислорода, предпочтение было отдано модели $P 4_{2} / n m c$.

Влияние температуры на теплоемкость $\mathrm{Bi}_{2} \mathrm{Pr}_{2} \mathrm{Ti}_{3} \mathrm{O}_{12}$ и $\mathrm{Bi}_{2} \mathrm{Nd}_{2} \mathrm{Ti}_{3} \mathrm{O}_{12}$ показано на рис. 2. Значения $C_{p}$ при росте температуры от 320 до $1000 \mathrm{~K}$ закономерно увеличиваются, а на зависимостях $C_{p}=f(T)$ нет разного рода экстремумов. По данным [37], на зависимости $C_{p}=f(T)$ для незамещенного титаната висмута в области сегнетоэлектрического фазового перехода при $943 \mathrm{~K}$ имеется экстремум, который для $\mathrm{Bi}_{2} \mathrm{Pr}_{2} \mathrm{Ti}_{3} \mathrm{O}_{12}$ и $\mathrm{Bi}_{2} \mathrm{Nd}_{2} \mathrm{Ti}_{3} \mathrm{O}_{12}$ не фиксируется. Подобное явление ранее наблюдалось при исследовании теплоемкости купратов $\mathrm{La}_{2-x} \mathrm{Sr}_{x} \mathrm{CuO}_{4}$ $(0 \leq x \leq 0.2)$ [38]. С увеличением концентрации $\mathrm{Sr}$ экстремум по кривой $C_{p}=f(T)$ смещается в зону более низких температур.

Экспериментальные результаты по теплоемкости $\mathrm{Bi}_{2} \mathrm{Pr}_{2} \mathrm{Ti}_{3} \mathrm{O}_{12}$ и $\mathrm{Bi}_{2} \mathrm{Nd}_{2} \mathrm{Ti}_{3} \mathrm{O}_{12}$ могут быть описаны урав-

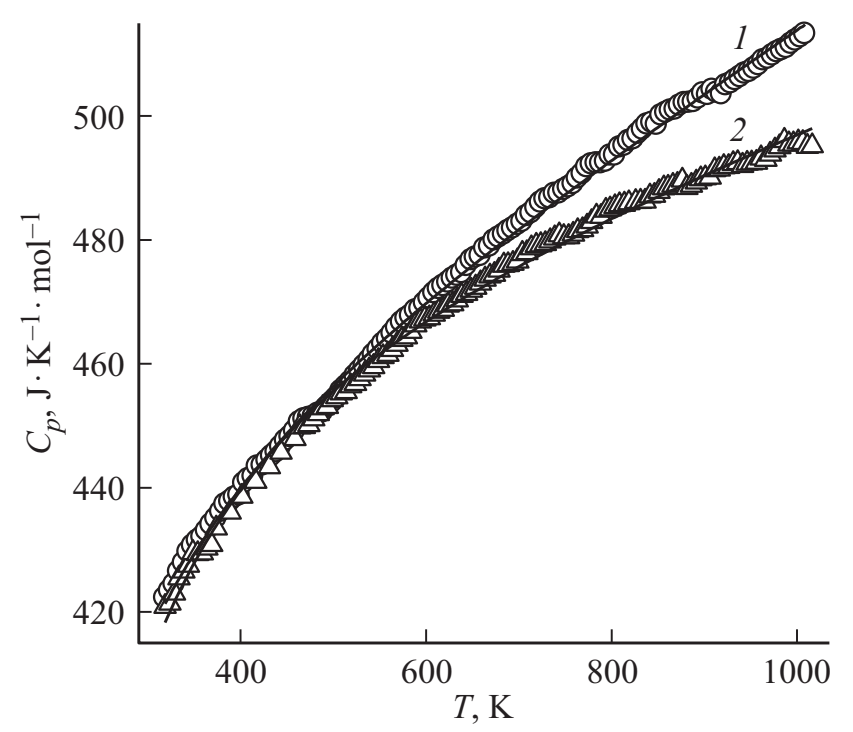

Рис. 2. Температурные зависимости молярной теплоемкости $\mathrm{Bi}_{2} \mathrm{Pr}_{2} \mathrm{Ti}_{3} \mathrm{O}_{12}(1) ; \mathrm{Bi}_{2} \mathrm{Nd}_{2} \mathrm{Ti}_{3} \mathrm{O}_{12}$ (2). 
Таблица 4. Термодинамические свойства $\mathrm{Bi}_{2} \mathrm{Pr}_{2} \mathrm{Ti}_{3} \mathrm{O}_{12}$ и $\mathrm{Bi}_{2} \mathrm{Nd}_{2} \mathrm{Ti}_{3} \mathrm{O}_{12}$

\begin{tabular}{|c|c|c|c|c|}
\hline$T, \mathrm{~K}$ & $\begin{array}{c}C_{p} \\
\mathrm{~J} \cdot \mathrm{K}^{-1} \cdot \mathrm{mol}^{-1}\end{array}$ & $\begin{array}{c}H^{\circ}(T)-H^{\circ}(320 \mathrm{~K}) \\
\mathrm{kJ} \cdot \mathrm{mol}^{-1}\end{array}$ & $\begin{array}{c}S^{\circ}(T)-S^{\circ}(320 \mathrm{~K}), \\
\mathrm{J} \cdot \mathrm{K}^{-1} \cdot \mathrm{mol}^{-1}\end{array}$ & $\begin{array}{c}-\Delta G / T^{*} \\
\mathrm{~J} \cdot \mathrm{K}^{-1} \cdot \mathrm{mol}^{-1}\end{array}$ \\
\hline \multicolumn{5}{|c|}{$\mathrm{Bi}_{2} \mathrm{Pr}_{2} \mathrm{Ti}_{3} \mathrm{O}_{12}$} \\
\hline $\begin{array}{r}320 \\
350 \\
400 \\
450 \\
500 \\
550 \\
600 \\
650 \\
700 \\
750 \\
800 \\
850 \\
900 \\
950 \\
1000\end{array}$ & $\begin{array}{l}421.7 \\
429.4 \\
440.0 \\
448.7 \\
456.4 \\
463.3 \\
469.8 \\
475.8 \\
481.7 \\
487.3 \\
492.8 \\
498.2 \\
503.5 \\
508.7 \\
513.9\end{array}$ & $\begin{array}{c}- \\
12.87 \\
34.51 \\
56.74 \\
79.70 \\
102.4 \\
125.7 \\
149.3 \\
173.3 \\
197.5 \\
222.0 \\
246.8 \\
271.8 \\
297.1 \\
322.7\end{array}$ & $\begin{array}{c}- \\
38.14 \\
96.19 \\
148.5 \\
196.2 \\
240.0 \\
280.6 \\
318.5 \\
354.0 \\
387.4 \\
419.0 \\
449.1 \\
477.7 \\
505.0 \\
531.2\end{array}$ & $\begin{array}{r}- \\
1.65 \\
9.91 \\
22.45 \\
37.48 \\
53.93 \\
71.15 \\
88.73 \\
106.4 \\
124.1 \\
141.5 \\
158.7 \\
175.7 \\
192.3 \\
208.6\end{array}$ \\
\hline \multicolumn{5}{|c|}{$\mathrm{Bi}_{2} \mathrm{Nd}_{2} \mathrm{Ti}_{3} \mathrm{O}_{12}$} \\
\hline $\begin{array}{r}320 \\
350 \\
400 \\
450 \\
500 \\
550 \\
600 \\
650 \\
700 \\
750 \\
800 \\
850 \\
900 \\
950 \\
1000\end{array}$ & $\begin{array}{l}418.7 \\
427.9 \\
439.6 \\
448.5 \\
455.6 \\
461.6 \\
466.8 \\
471.5 \\
475.7 \\
479.7 \\
483.4 \\
487.0 \\
490.4 \\
493.7 \\
497.0\end{array}$ & $\begin{array}{c}- \\
12.70 \\
34.41 \\
56.62 \\
79.23 \\
102.2 \\
125.4 \\
148.8 \\
172.5 \\
196.4 \\
220.5 \\
244.7 \\
269.2 \\
293.8 \\
318.5\end{array}$ & $\begin{array}{c}- \\
37.94 \\
95.89 \\
148.2 \\
195.8 \\
239.5 \\
279.9 \\
317.5 \\
352.6 \\
385.5 \\
416.6 \\
446.0 \\
473.7 \\
500.6 \\
526.0\end{array}$ & $\begin{array}{r}- \\
1.64 \\
9.87 \\
22.38 \\
37.38 \\
53.80 \\
70.98 \\
88.51 \\
106.1 \\
123.7 \\
141.0 \\
158.1 \\
174.9 \\
191.3 \\
207.4\end{array}$ \\
\hline
\end{tabular}

Примечание. ${ }^{*} \Delta G / T=\left[H^{\circ}(T)-H^{\circ}(320 \mathrm{~K})\right] / T-\left[S^{\circ}(T)-S^{\circ}(320 \mathrm{~K})\right]$.

нением Майера-Келли [39]:

$$
C_{p}=a+b T-c T^{-2},
$$

имеющим, соответственно, для исследованных титанатов висмута следующий вид $\left(\mathrm{J} \cdot \mathrm{K}^{-1} \cdot \mathrm{mol}^{-1}\right)$

$$
\begin{aligned}
C_{p}=(419.9 \pm 1.1) & +(96.90 \pm 1.2) \cdot 10^{-3} T \\
& -(29.99 \pm 1.05) \cdot 10^{5} T^{-2}, \\
C_{p}=(417.1 \pm 1.1) & +(54.55 \pm 1.2) \cdot 10^{-3} T \\
& -(46.90 \pm 1.11) \cdot 10^{5} T^{-2} .
\end{aligned}
$$

Коэффициенты корреляции для уравнений (2) и (3) равны 0.9979 и 0.9982, а максимальные отклонения экспериментальных точек от сглаживающих кривых 1.17 и $1.23 \%$ соответственно.

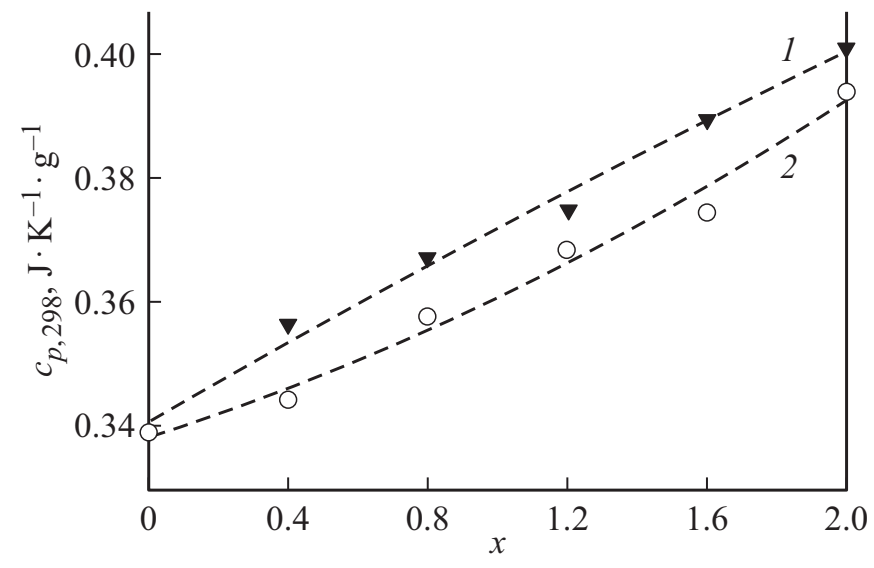

Рис. 3. Влияние состава твердых растворов $\mathrm{Bi}_{2} \mathrm{Pr}_{2-x} \mathrm{Ti}_{3} \mathrm{O}_{12}$ (1) и $\mathrm{Bi}_{2} \mathrm{Nd}_{2-x} \mathrm{Ti}_{3} \mathrm{O}_{12}(2)$ и на их теплоемкость. 
Сравнить полученные результаты по теплоемкости $\mathrm{Bi}_{2} \mathrm{Pr}_{2} \mathrm{Ti}_{3} \mathrm{O}_{12}$ и $\mathrm{Bi}_{2} \mathrm{Nd}_{2} \mathrm{Ti}_{3} \mathrm{O}_{12}$ с данными других авторов не представлялось возможным вследствие их отсутствия. Учитывая это, нами проведено изучение влияния состава твердых растворов на их теплоемкость (рис. 3). Чтобы не учитывать различие молярных масс, приведены значения удельных теплоемкостей $c_{p}$. Видно, что с ростом концентрации редкоземельных элементов значения $c_{p}$ при $298 \mathrm{~K}$ закономерно увеличиваются. Это, по-видимому, может свидетельствовать о достоверности полученных значений теплоемкости для $\mathrm{Bi}_{2} \mathrm{Pr}_{2} \mathrm{Ti}_{3} \mathrm{O}_{12}$ и $\mathrm{Bi}_{2} \mathrm{Nd}_{2} \mathrm{Ti}_{3} \mathrm{O}_{12}$.

С использованием уравнений (2) и (3) по известным термодинамическим соотношениям рассчитаны термодинамические функции замещенных титанатов висмута. Эти результаты приведены в табл. 4.

\section{4. Заключение}

Твердофазным синтезом получены замещенные титанаты $\mathrm{Bi}_{2} \mathrm{Pr}_{2} \mathrm{Ti}_{3} \mathrm{O}_{12}$ и $\mathrm{Bi}_{2} \mathrm{Nd}_{2} \mathrm{Ti}_{3} \mathrm{O}_{12}$. Определена кристаллическая структура и исследовано влияние температуры $(320-1000 \mathrm{~K})$ на их теплоемкость. С использованием экспериментальных данных $C_{p}=f(T)$ рассчитаны термодинамические функции.

\section{Благодарности}

Авторы выражают благодарность Красноярскому региональному центру коллективного пользования ФИЦ КНЦ СО РАН.

\section{Финансирование работы}

Работа выполнена при частичной финансовой поддержке в рамках государственного задания на науку ФГАОУ ВО „Сибирский федеральный университет“, номер проекта FSRZ-2020-0013.

\section{Конфликт интересов}

Авторы заявляют, что у них нет конфликта интересов.

\section{Список литературы}

[1] M.S. Tomar, R.E. Melgarejo, A. Hidalgo, S.B. Mazumder, R.S. Katiyar. Appl. Phys. Lett. 33, 2, 341 (2003).

[2] N. Pavlović, D. Kanco, K.M. Szécsénji, V.V. Srdié. Proc. Appl. Ceram. 3, 1-2, 88 (2009).

[3] А.И. Клындюк, А.А. Глинская, Е.А. Чижова, Л.А. Башкиров. Огнеупоры и техн. керам. 1-2, 29 (2017).

[4] C. Yang, Z. Wang, D. Pan, J. Han, Q. Li, J. Wang. Sur. Rev. Lett. 11, 6, 503 (2004).

[5] Y.-M. Kan, G.-J. Zhang, P.-L. Wang, Y.-B. Cheng. J. Eur. Ceram. Soc. 28, 1641 (2008).
[6] Y. Wang, N. Zhao, M. Zhang, X. Zhao. J. Wuhan Univer. Thechnol.-Mater. Sci. 25, 5, 743 (2010).

[7] M. Iwata, A. Toya, R. Aoyagi, M. Maeda, Y. Ishibashi. Jpn. J. Appl. Phys. 47, 9, 7749 (2008).

[8] Z.X. Cheng, X.L. Wang, S.X. Dou, K. Ozawa, H. Kimura. Appl. Phys. Lett. 90, 222902-1 (2007).

[9] U. Chon, K.-B. Kim, H.M. Jang, G.-C. Yi. Appl. Phys. Lett. 79, 19, 3137 (2001).

[10] M.E. Villafuerte-Castrejon, F. Camacho-Alanis, F. González, A. Ibarra-Palos, G. González, L. Fuentes, L. Bucio. J. Eur. Ceram. Soc. 27, 545 (2007).

[11] F. Yang, B. Jia, T. Wei, C. Zhao, Q. Zhou, Z. Li, M. Du, M. Wang, Y. Liu, C. Xie. Inorg. Chem. Front. 6, 2756 (2019).

[12] A. Huanosta-Tera, R. Castañeda-Guzman, J.L. Pineda-Flores. Mater. Res. Bull. 38, 1073 (2003).

[13] J.L. Pineda-Flores, E. Chavira, J. Reyes-Garga, A.M. González, A. Huanosta-Tera. J. Eur. Ceram. Soc. 23, 839 (2003).

[14] S.J. Kim, C. Moriyoshi, S. Kimura, Y. Kuroiwa, K. Koto, M. Takata, Y. Noguchi, M. Miyayama. Appl. Phys. Lett. 91, 062913-1 (2007).

[15] А.Н. Калинкин, Е.М. Кожбахтеев, А.Е. Поляков, В.М. Скориков. Неорган. материалы 49, 10, 1113 (2013).

[16] S.A. Ivanov, T. Sarkar, E.A. Fortalnova, E.D. Politova, S.Yu. Stefanovich, M.G. Safronenko, P. Nordbland, R. Mathieu. J. Mater. Sci.: Mater. Electron. 28, 7692 (2017).

[17] W. Gong, R. Zhang. J. Alloys Compd. 548, 216 (2013).

[18] М.А. Петрова, А.С. Новикова, Р.Г. Гребенщиков. ДАН CCCP. 246, 1, 121 (1979).

[19] М.А. Петрова, А.С. Новикова, Р.Г. Гребенщиков. Изв. АН СССР. Неорган. материалы 18, 4, 700 (1982).

[20] Š. Kunej, D. Suvorov. J. Am. Ceram. Soc. 91, 10, 3472 (2008).

[21] J. Takahashi, K. Kageyama, T. Hayashi. Jpn. J. Appl. Phys. 30, 9B, 2354 (1991).

[22] G. Garcia-Martinez, L.G. Martinez-Gonzalez, J.I. EscalanteGarcia, A.F. Fuentes. Powder Technol. 152, 72 (2005).

[23] Л.Н. Комиссарова, В.М. Шацкий, Г.Я. Пушкина, Л.Г. Щербакова, Л.Г. Мамсурова, Г.Е. Суханова. Соединения редкоземельных элементов. Карбонаты, силикаты, нитраты, титанаты. Наука, М. (1984). 235 с.

[24] P. Kostantinov, K. Krezhov, E. Sváb, G. Mészáros, Gy. Török. Phys. B. 276-278, 260 (2000).

[25] X. Li, W. Wu, F. Liu, Y. Li, P. Si, H. Ge. Mater. Lett. 118, 24 (2014).

[26] Л.В. Морозова. Неорган. материалы 55, 3, 323 (2019).

[27] В.М. Денисов, Л.Т. Денисова, Л.А. Иртюго, В.С. Биронт. ФТT 52, 7, 1274 (2010).

[28] Л.Т. Денисова, Л.А. Иртюго, Ю.Ф. Каргин, В.В. Белецкий, В.М. Денисов. Неорган. материалы 55, 3, 516 (2019).

[29] Bruker AXS TOPAS V4: General profile and structure analysis software for powder diffraction data. User's Manual. Bruker AXS, Karlsruhe, Germany (2008).

[30] Н.А. Ломанова, М.В. Томкович, В.Л. Уголков, В.В. Гусаров. ЖПХ 90, 6, 673 (2017).

[31] C. Long, Q. Chang, H. Fan. Sci. Rep. 7, 4193 (2017).

[32] M. Takahashi, Y. Noguchi, M. Miyayama. Jpn. J. Appl. Phys. 41, 7053 (2002).

[33] N.C. Hyatt, J.A. Hriljac, T.P. Comyn. Mater. Res. Bull. 38, 837 (2003). 
[34] M. Takahashi, Y. Noguchi, M. Miyayama. J. Ceram. Proc. Res. 6, 4, 281 (2005).

[35] Gopalakrishnan, T. Sivakumar, K. Ramesha, V. Thangadurai, G.N. Subbanna. J. Am. Chem. Soc. 122, 6237 (2000).

[36] C. Capillas, E.S. Tasci, G. de la Flor, D. Orobengoa, J.M. PerezMato, M.I. Aroyo. Z. Kristal. Mater. 226, 2, 186 (2011).

[37] Л.Т. Денисова, Ю.Ф. Каргин, Л.Г. Чумилина, Н.В. Белоусова, В.М. Денисов. Неорган. материалы 56, 6, 630 (2020).

[38] K. Sun, J.H. Cho, F.C. Chou, W.C. Lee, L.L. Miller, D.C. Johnston. Phys. Rev. B. 43, 239 (1991).

[39] C.G. Maier, K.K. Kelley. J. Am. Chem. Soc. 54, 3234 (1932).

Редактор Т.Н. Василевская 